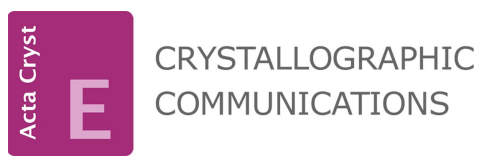

ISSN 2056-9890

Received 14 February 2015

Accepted 24 February 2015

Edited by $\mathrm{H}$. Stoeckli-Evans, University of Neuchâtel, Switzerland

Keywords: crystal structure; PTP1B; inhibitor; 1,2,5-thiadiazolidin-3-one 1,1-dioxide; hydrogen bonding

CCDC reference: 1051176 Supporting information: this article has supporting information at journals.iucr.org/e
Crystal structure of 5-\{4'-[(2-\{2-[2-(2-ammonioethoxy)ethoxy]ethoxy\}ethyl)carbamoyl]-4-methoxy[1,1'-biphenyl]-3-yl\}-3-oxo-1,2,5-thiadiazolidin-2ide 1,1-dioxide: a potential inhibitor of the enzyme protein tyrosine phosphatase 1B (PTP1B)

Kasi Viswanatharaju Ruddraraju, Roman Hillebrand, Charles L. Barnes and Kent S. Gates*

125 Chemistry Bldg, University of Missouri Columbia, MO 65211, USA. *Correspondence e-mail: gatesk@missouri.edu

The title compound, $\mathrm{C}_{24} \mathrm{H}_{32} \mathrm{~N}_{4} \mathrm{O}_{8} \mathrm{~S}$, (I), crystallizes as a zwitterion. The terminal amine $\mathrm{N}$ atom of the [(2-\{2-[2-(2-ammonioethoxy)ethoxy]ethoxy\}ethyl)carbamoyl] side chain is protonated, while the 1,2,5-thiadiazolidin-3-one 1,1-dioxide $\mathrm{N}$ atom is deprotonated. The side chain is turned over on itself with an intramolecular $\mathrm{N}-\mathrm{H} \cdots \mathrm{O}$ hydrogen bond. The 1,2,5-thiadiazolidin-3-one 1,1-dioxide ring has an envelope conformation with the aryl-substituted $\mathrm{N}$ atom as the flap. Its mean plane is inclined by $62.87(8)^{\circ}$ to the aryl ring to which it is attached, while the aryl rings of the biphenyl unit are inclined to one another by $20.81(8)^{\circ}$. In the crystal, molecules are linked by $\mathrm{N}-\mathrm{H} \cdots \mathrm{O}$ and $\mathrm{N}-\mathrm{H} \cdots \mathrm{N}$ hydrogen bonds, forming slabs lying parallel to (010). Within the slabs there are $\mathrm{C}-\mathrm{H} \cdots \mathrm{O}$ and $\mathrm{C}-\mathrm{H} \cdots \mathrm{N}$ hydrogen bonds and $\mathrm{C}-\mathrm{H} \cdots \pi$ interactions present.

\section{Chemical context}

A variety of 5-aryl-1,2,5-thiadiazolidin-3-one 1,1-dioxides have been developed as inhibitors of the enzyme protein tyrosine phosphatase 1B (PTP1B) (Combs, 2010). In this capacity, the 5-aryl-1,2,5-thiadiazolidin-3-one 1,1-dioxide core serves as a structural mimic of the phosphoryl tyrosine unit that is present in the endogenous substrates of the enzyme. The parent compound, 5-phenyl-1,2,5-thiadiazolidin-3-one 1,1-dioxide 1 (Fig. 1), is a rather weak inhibitor of PTP1B, displaying a $\mathrm{K}_{\mathrm{i}}$ value of approximately $2 \mathrm{~m} M$ (Black et al., 2005). Docking studies predicted that this compound must bind to the enzyme active site in a conformation where the planes of the 1,2,5-thiadiazolidin-3-one 1,1-dioxide and aryl<smiles>[R]c1ccccc1N1CC(=O)NS1(=O)=O</smiles>

$1 \mathrm{R}=\mathrm{H}$

$2 \mathrm{R}=\mathrm{CH}_{3}$ $3 \mathrm{R}=\mathrm{OCH}_{3}$<smiles>COc1ccc(-c2ccccc2)cc1N1CC(=O)NS1(=O)=O</smiles>

Figure 1

The parent compound $\mathbf{1}$ and related compounds. 
rings are twisted, rather than co-planar (Black et al., 2005). It was further anticipated that installation of substituents such as methyl or methoxy groups on the aryl ring at the position ortho to the 1,2,5-thiadiazolidin-3-one 1,1-dioxide substituent would bias the conformation of the free ligand toward the twisted form, thus serving to 'pre-organize' the compounds for binding to the enzyme active site (Black et al., 2005). Indeed, compounds 2 and 3 ( $\mathrm{K}_{\mathrm{i}}$ values of 100 and $70 \mu M$, respectively) display substantially higher affinities for PTP1B than does $\mathbf{1}$ (Black et al., 2005). X-ray crystal structure analysis confirmed the twisted conformation of the 1,2,5-thiadiazolidin-3-one 1,1-dioxide and aryl ring systems in the protein-ligand cocrystal structure of $\mathbf{4}$ bound to PTP1B (Black et al., 2005). The planes of these two rings are nearly perpendicular in the protein-ligand complex (dihedral angle of $c a 88^{\circ}$, see: pdb code $2 \mathrm{bgd}$ ). The ability of methyl and methoxy substituents to favor the twisted relationship between the 1,2,5-thiadiazolidin-3-one 1,1-dioxide and aryl rings in compounds like $\mathbf{2}$ and $\mathbf{3}$ has been studied computationally and the twisted relationship of these rings has been experimentally observed in the protein-ligand co-crystal structure of $\mathbf{4}$ with the enzyme PTP1B. However, to the best of our knowledge no crystal structures of free 5-aryl-1,2,5-thiadiazolidin-3-one 1,1-dioxides have been published. Herein, we describe the crystal structure of the title compound (I), shown in the scheme below, a derivative of compound 4 .

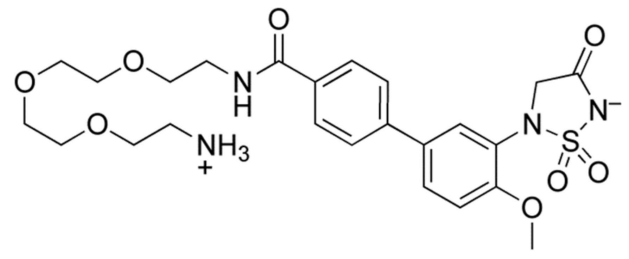

\section{Structural commentary}

The title compound (I), crystallized as a zwitterion (Fig. 2). The terminal amine $\mathrm{N}$ atom, $\mathrm{N} 4$, is protonated and the 1,2,5-

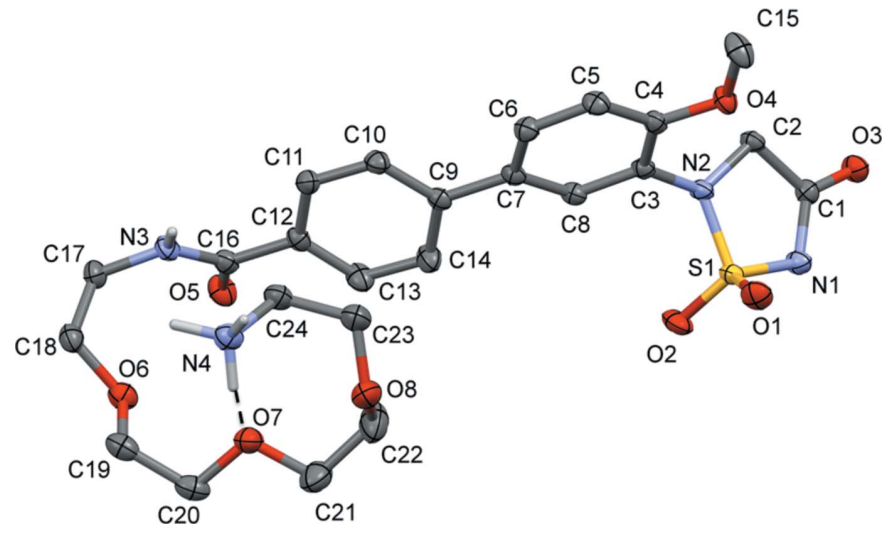

Figure 2

A view of the molecular structure of the title compound (I), showing the atom labelling. Displacement ellipsoids are drawn at the $50 \%$ probability level. The intramolecular $\mathrm{N}-\mathrm{H} \cdots \mathrm{O}$ hydrogen bond is shown as a dashed line (see Table 1 for details) and $\mathrm{C}$-bound $\mathrm{H}$ atoms have been omitted for clarity.
Table 1

Hydrogen-bond geometry $\left(\AA,^{\circ}\right)$.

$C g 1$ is the centroid of the $\mathrm{C} 3-\mathrm{C} 8$ ring.

\begin{tabular}{|c|c|c|c|c|}
\hline$D-\mathrm{H} \cdots A$ & $D-\mathrm{H}$ & $\mathrm{H} \cdots A$ & $D \cdots A$ & $D-\mathrm{H} \cdots A$ \\
\hline $\mathrm{N} 3-\mathrm{H} 1 N 3 \cdots \mathrm{O} 3^{\mathrm{i}}$ & $0.82(2)$ & $2.22(3)$ & $3.012(2)$ & $161(2)$ \\
\hline $\mathrm{N} 4-\mathrm{H} 1 N 4 \cdots \mathrm{O} 1^{\mathrm{ii}}$ & $0.93(3)$ & $2.29(3)$ & $3.010(2)$ & $133(2)$ \\
\hline $\mathrm{N} 4-\mathrm{H} 1 N 4 \ldots \mathrm{O} 7$ & $0.93(3)$ & $2.49(3)$ & $3.106(2)$ & $124(2)$ \\
\hline $\mathrm{N} 4-\mathrm{H} 2 N 4 \cdots \mathrm{N} 1^{\mathrm{i}}$ & $1.03(3)$ & $1.82(3)$ & $2.821(2)$ & $163(2)$ \\
\hline $\mathrm{N} 4-\mathrm{H} 3 N 4 \cdots \mathrm{O} 6^{\mathrm{iii}}$ & $0.98(3)$ & 1.99 (3) & $2.942(2)$ & $162(3)$ \\
\hline $\mathrm{C} 2-\mathrm{H} 2 B \cdots \mathrm{O} 3^{\mathrm{iv}}$ & 0.99 & 2.30 & 3.267 (2) & 166 \\
\hline $\mathrm{C} 18-\mathrm{H} 18 A \cdots \mathrm{N} 1^{\mathrm{i}}$ & 0.99 & 2.57 & $3.545(2)$ & 168 \\
\hline $\mathrm{C} 22-\mathrm{H} 22 A \cdots \mathrm{O} 8^{\mathrm{ii}}$ & 0.99 & 2.63 & $3.343(3)$ & 129 \\
\hline $\mathrm{C} 24-\mathrm{H} 24 A \cdots \mathrm{O} 5^{\mathrm{iii}}$ & 0.99 & 2.58 & $3.298(2)$ & 129 \\
\hline $\mathrm{C} 21-\mathrm{H} 21 B \cdots C g 1^{\mathrm{ii}}$ & 0.99 & 2.70 & $3.555(2)$ & 165 \\
\hline
\end{tabular}

Symmetry codes: (i) $x, y, z+1$; (ii) $-x+1,-y+1,-z+1$; (iii) $x+1, y$, $z$; (iv) $-x+1,-y+2,-z$.

thiadiazolidin-3-one 1,1-dioxide nitrogen atom, N1, is deprotonated. The [(2-\{2-[2-(2-ammonioethoxy)ethoxy]ethoxy\}ethyl)carbamoyl] side chain is folded over on itself with an intramolecular $\mathrm{N}-\mathrm{H} \cdots \mathrm{O}$ hydrogen bond involving the ammonium group, $\mathrm{N} 4$, and an ether $\mathrm{O}$ atom, $\mathrm{O} 7$ (Table 1 and Fig. 2). The aryl rings of the biphenyl unit ( $\mathrm{C} 3-\mathrm{C} 8$ and $\mathrm{C} 9$ C14) are inclined to one another by $20.81(8)^{\circ}$. The $1,2,5-$ thiadiazolidin-3-one 1,1-dioxide ring (S1/N1/N2/C1/C2) has a shallow envelope conformation with nitrogen atom $\mathrm{N} 2$ as the flap. Its mean plane is inclined to the benzene ring to which it is attached $(\mathrm{C} 3-\mathrm{C} 8)$ by $62.87(8)^{\circ}$. This twisted relationship between the planes of the 1,2,5-thiadiazolidin-3-one 1,1-dioxide and aryl rings is substantially less than that seen in the protein-ligand co-crystal structure of $\mathbf{4}$ bound to PTP1B (Black et al., 2005), where these two rings are nearly perpendicular to one another with a dihedral angle of $c a 88^{\circ}$ (see: Protein Data Bank entry: code 2bgd).

\section{Supramolecular features}

In the crystal of (I), molecules are linked by $\mathrm{N}-\mathrm{H} \cdots \mathrm{O}$ and $\mathrm{N}-\mathrm{H} \cdots \mathrm{N}$ hydrogen bonds, forming slabs lying parallel to the ac plane (Fig. 3 and Table 1). Within the slabs there are also

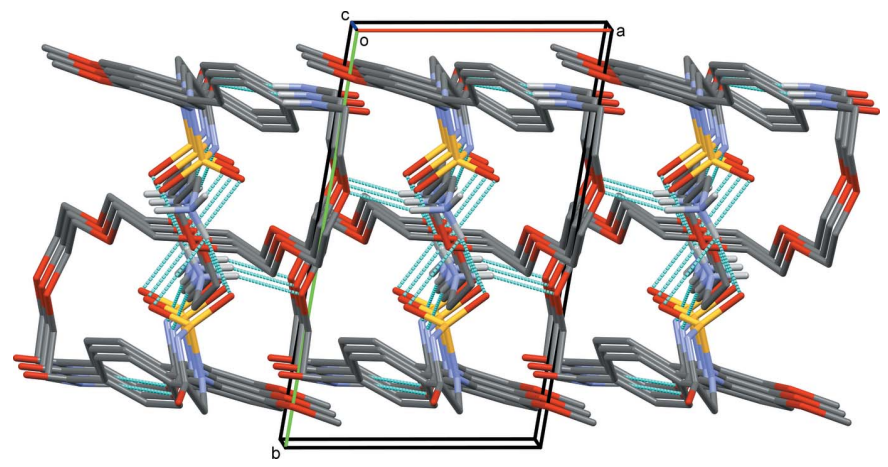

Figure 3

A view along the $c$ axis of the crystal packing of the title compound. The $\mathrm{N}-\mathrm{H} \cdots \mathrm{O}$ and $\mathrm{N}-\mathrm{H} \cdots \mathrm{O}$ hydrogen bonds are shown as dashed lines (see Table 1 for details) and $\mathrm{C}$-bound $\mathrm{H}$ atoms have been omitted for clarity. 
Table 2

Experimental details.

\begin{tabular}{ll}
\hline Crystal data & \\
Chemical formula & $\mathrm{C}_{24} \mathrm{H}_{32} \mathrm{~N}_{4} \mathrm{O}_{8} \mathrm{~S}$ \\
$M_{\mathrm{r}}$ & 536.59 \\
Crystal system, space group & Triclinic, $P \overline{1}$ \\
Temperature $(\mathrm{K})$ & 100 \\
$a, b, c(\AA)$ & $7.3483(2), 12.2233(3), 13.9847(4)$ \\
$\alpha, \beta, \gamma\left({ }^{\circ}\right)$ & $95.323(1), 90.281(2), 99.802(1)$ \\
$V\left(\AA^{3}\right)$ & $1232.16(6)$ \\
$Z$ & 2 \\
Radiation type & $\mathrm{Cu} \mathrm{K \alpha}$ \\
$\mu\left(\mathrm{mm}^{-1}\right)$ & 1.67 \\
Crystal size $(\mathrm{mm})$ & $0.15 \times 0.15 \times 0.02$ \\
& \\
Data collection & Bruker APEXII CCD area \\
Diffractometer & detector \\
& Multi-scan $(S A D A B S ;$ Bruker, \\
Absorption correction & $2008)$ \\
& $0.89,0.97$ \\
$T_{\text {min }}, T_{\max }$ & $15014,4539,4292$ \\
No. of measured, independent and & \\
$\quad$ observed $[I>2 \sigma(I)]$ reflections & 0.017 \\
$R_{\text {int }}$ & 0.617 \\
$(\text { sin } \theta / \lambda)_{\text {max }}\left(\AA^{-1}\right)$ & \\
Refinement & \\
$R\left[F^{2}>2 \sigma\left(F^{2}\right)\right], w R\left(F^{2}\right), S$ & $0.039,0.111,1.03$ \\
No. of reflections & 4539 \\
No. of parameters & 351 \\
H-atom treatment & H atoms treated by a mixture of \\
& independent and constrained \\
$\Delta \rho_{\text {max }}, \Delta \rho_{\text {min }}\left(\mathrm{e} \AA^{-3}\right)$ & refinement \\
\hline & $0.56,-0.33$ \\
\hline
\end{tabular}

Computer programs: APEX2 and SAINT (Bruker, 2008), SHELXS2013 (Sheldrick, 2008), SHELXL2013 (Sheldrick, 2015) and Mercury (Macrae et al., 2008).

$\mathrm{C}-\mathrm{H} \cdots \mathrm{O}$ and $\mathrm{C}-\mathrm{H} \cdots \mathrm{N}$ hydrogen bonds and $\mathrm{C}-\mathrm{H} \cdots \pi$ interactions present reinforcing the two-dimensional structure (Table 1).

\section{Database survey}

A search of the Cambridge Structural Database (Version 5.36; Groom \& Allen, 2014) revealed no crystal structures of free 5-aryl-1,2,5-thiadiazolidin-3-one 1,1-dioxides. It did reveal the presence of five 1,2,5-thiadiazolidin-3-one 1,1-dioxide compounds substituted at the $\mathrm{N}$ atom in the 2-position. In the majority of these compounds, the five-membered 1,2,5-thiadiazolidine rings also have envelope conformations, with the $\mathrm{N}$ atom in the 5-position, as in compound (I), as the flap.

\section{Synthesis and crystallization}

The title compound was synthesized by amide bond formation between tert-butyl (2-\{2-[2-(2-aminoethoxy)ethoxy]ethoxy\}ethyl)carbamate and $3^{\prime}$-(1,1-dioxido-4-oxo-1,2,5-thiadiazolidin-2-yl)-4'-methoxy-[1,1'-biphenyl]-4-carboxylic acid via (benzotriazol-1-yloxy)tris(dimethylamino)phosphonium hexafluorophosphate. The precursors were synthesized according to published procedures (Black et al., 2005; Schwabacher et al., 1998). Full synthetic details will be published elsewhere. Single crystals of the title compound (I) were obtained by slow evaporation of a solution of (I) in methanol.

\section{Refinement details}

Crystal data, data collection and structure refinement details are summarized in Table 2. The $\mathrm{N}$-bound $\mathrm{H}$ atoms were located in a difference Fourier map and freely refined. The Cbound $\mathrm{H}$ atoms were included in calculated positions and treated as riding: $\mathrm{C}-\mathrm{H}=0.95-0.99 \AA$ with $U_{\text {iso }}(\mathrm{H})=$ $1.5 U_{\text {eq }}(\mathrm{C})$ for methyl $\mathrm{H}$ atoms and $=1.2 U_{\text {eq }}(\mathrm{C})$ for other $\mathrm{H}$ atoms.

\section{Acknowledgements}

We are grateful to the National Institutes of Health (CA 100757) for partial support of this work.

\section{References}

Black, E., Breed, J., Breeze, A. L., Embrey, K., Garcia, R., Gero, T. W., Godfrey, L., Kenny, P. W., Morley, A. D., Minshull, C. A., Pannifer, A. D., Read, J., Rees, A., Russell, D. J., Toader, D. \& Tucker, J. (2005). Bioorg. Med. Chem. Lett. 15, 2503-2507.

Bruker (2008). APEX2, SAINT and SADABS. Bruker AXS Inc., Madison, Wisconsin, USA.

Combs, A. P. (2010). J. Med. Chem. 53, 2333-2344.

Groom, C. R. \& Allen, F. H. (2014). Angew. Chem. Int. Ed. 53, 662671.

Macrae, C. F., Bruno, I. J., Chisholm, J. A., Edgington, P. R., McCabe, P., Pidcock, E., Rodriguez-Monge, L., Taylor, R., van de Streek, J. \& Wood, P. A. (2008). J. Appl. Cryst. 41, 466-470.

Schwabacher, A. W., Lane, J. W., Schiesher, M. W., Leigh, K. M. \& Johnson, C. W. (1998). J. Org. Chem. 63, 1727-1729.

Sheldrick, G. M. (2008). Acta Cryst. A64, 112-122.

Sheldrick, G. M. (2015). Acta Cryst. C71, 3-8. 


\section{supporting information}

Acta Cryst. (2015). E71, 336-338 [doi:10.1107/S2056989015003850]

Crystal structure of 5-\{4'-[(2-\{2-[2-(2-ammonioethoxy)ethoxy] ethoxy\}ethyl)carbamoyl]-4-methoxy-[1,1'-biphenyl]-3-yl\}-3-oxo-1,2,5-thiadiazolidin-2-ide 1,1-dioxide: a potential inhibitor of the enzyme protein tyrosine phosphatase 1 B (PTP1B)

Kasi Viswanatharaju Ruddraraju, Roman Hillebrand, Charles L. Barnes and Kent S. Gates

Computing details

Data collection: APEX2 (Bruker, 2008); cell refinement: SAINT (Bruker, 2008); data reduction: SAINT (Bruker, 2008); program(s) used to solve structure: SHELXS2013 (Sheldrick, 2008); program(s) used to refine structure: SHELXL2013 (Sheldrick, 2015); molecular graphics: Mercury (Macrae et al., 2008); software used to prepare material for publication: SHELXL2013 (Sheldrick, 2015).

5-\{4'-[(2-\{2-[2-(2-Ammonioethoxy)ethoxy] ethoxy\}ethyl) carbamoyl]-4-methoxy-[1,1'-biphenyl]-3-yl\}-3-oxo-1,2,5thiadiazolidin-2-ide 1,1-dioxide

Crystal data

$\mathrm{C}_{24} \mathrm{H}_{32} \mathrm{~N}_{4} \mathrm{O}_{8} \mathrm{~S}$

$M_{r}=536.59$

Triclinic, $P \overline{1}$

$a=7.3483$ (2) $\AA$

$b=12.2233$ (3) $\AA$

$c=13.9847$ (4) $\AA$

$\alpha=95.323(1)^{\circ}$

$\beta=90.281(2)^{\circ}$

$\gamma=99.802(1)^{\circ}$

$V=1232.16(6) \AA^{3}$

Data collection

Bruker APEXII CCD area-detector diffractometer

Radiation source: Incoatec microfocus $\mathrm{Cu}$ tube $\omega$ and phi scans

Absorption correction: multi-scan

(SADABS; Bruker, 2008)

$T_{\min }=0.89, T_{\max }=0.97$

15014 measured reflections

Refinement

Refinement on $F^{2}$

Least-squares matrix: full

$R\left[F^{2}>2 \sigma\left(F^{2}\right)\right]=0.039$
$Z=2$

$F(000)=568$

$D_{\mathrm{x}}=1.446 \mathrm{Mg} \mathrm{m}^{-3}$

$\mathrm{Cu} K \alpha$ radiation, $\lambda=1.54178 \AA$

Cell parameters from 8971 reflections

$\theta=3.2-71.7^{\circ}$

$\mu=1.67 \mathrm{~mm}^{-1}$

$T=100 \mathrm{~K}$

Plate, colourless

$0.15 \times 0.15 \times 0.02 \mathrm{~mm}$

4539 independent reflections

4292 reflections with $I>2 \sigma(I)$

$R_{\text {int }}=0.017$

$\theta_{\max }=72.1^{\circ}, \theta_{\min }=3.2^{\circ}$

$h=-8 \rightarrow 7$

$k=-15 \rightarrow 15$

$l=-16 \rightarrow 16$

$w R\left(F^{2}\right)=0.111$

$S=1.03$

4539 reflections 
351 parameters

0 restraints

Hydrogen site location: mixed

$\mathrm{H}$ atoms treated by a mixture of independent and constrained refinement

$$
\begin{aligned}
& w=1 /\left[\sigma^{2}\left(F_{\mathrm{o}}^{2}\right)+(0.0647 P)^{2}+0.8661 P\right] \\
& \text { where } P=\left(F_{\mathrm{o}}^{2}+2 F_{\mathrm{c}}^{2}\right) / 3 \\
& (\Delta / \sigma)_{\max }<0.001 \\
& \Delta \rho_{\max }=0.56 \mathrm{e} \AA^{-3} \\
& \Delta \rho_{\min }=-0.33 \mathrm{e} \AA^{-3}
\end{aligned}
$$

\section{Special details}

Geometry. All e.s.d.'s (except the e.s.d. in the dihedral angle between two 1.s. planes) are estimated using the full covariance matrix. The cell e.s.d.'s are taken into account individually in the estimation of e.s.d.'s in distances, angles and torsion angles; correlations between e.s.d.'s in cell parameters are only used when they are defined by crystal symmetry.

\begin{tabular}{|c|c|c|c|c|}
\hline & $x$ & $y$ & $z$ & $U_{\text {iso }} * / U_{\text {eq }}$ \\
\hline S1 & $0.52151(5)$ & $0.68948(3)$ & $0.09933(3)$ & $0.01945(13)$ \\
\hline $\mathrm{O} 1$ & 0.65998 (19) & $0.61868(11)$ & $0.09811(10)$ & $0.0305(3)$ \\
\hline $\mathrm{O} 2$ & $0.35802(18)$ & $0.64660(11)$ & $0.14874(10)$ & $0.0317(3)$ \\
\hline $\mathrm{O} 3$ & $0.52042(18)$ & $0.86988(10)$ & $-0.09372(9)$ & $0.0266(3)$ \\
\hline $\mathrm{O} 4$ & $0.97456(17)$ & $0.88464(10)$ & $0.17601(9)$ & $0.0242(3)$ \\
\hline O5 & $-0.07338(17)$ & $0.81837(11)$ & $0.69084(9)$ & $0.0296(3)$ \\
\hline O6 & $-0.04449(18)$ & $0.63027(11)$ & $0.85611(9)$ & $0.0279(3)$ \\
\hline O7 & $0.16486(19)$ & $0.49974(10)$ & $0.73225(10)$ & $0.0305(3)$ \\
\hline O8 & $0.5123(2)$ & $0.49283(11)$ & $0.63370(10)$ & $0.0359(3)$ \\
\hline N1 & $0.4761(2)$ & $0.71885(12)$ & $-0.00657(10)$ & 0.0239 \\
\hline $\mathrm{N} 2$ & $0.6032(2)$ & $0.81729(11)$ & $0.14491(10)$ & $0.0221(3)$ \\
\hline N3 & $0.1468(2)$ & $0.84118(12)$ & $0.80862(11)$ & 0.0232 \\
\hline H1N3 & $0.256(3)$ & $0.8427(18)$ & $0.8228(16)$ & $0.027(6)^{*}$ \\
\hline N4 & $0.5520(3)$ & $0.58747(14)$ & $0.82658(12)$ & $0.0291(3)$ \\
\hline H1N4 & $0.474(4)$ & $0.520(3)$ & $0.812(2)$ & $0.052(8)^{*}$ \\
\hline $\mathrm{H} 2 \mathrm{~N} 4$ & $0.510(3)$ & $0.621(2)$ & 0.8909 (19) & $0.041(6)^{*}$ \\
\hline H3N4 & $0.685(5)$ & $0.585(2)$ & $0.831(2)$ & $0.058(8)^{*}$ \\
\hline $\mathrm{C} 1$ & $0.5371(2)$ & $0.82684(14)$ & $-0.01819(12)$ & $0.0206(3)$ \\
\hline $\mathrm{C} 2$ & $0.6324(2)$ & $0.89346(13)$ & $0.07032(11)$ & $0.0198(3)$ \\
\hline $\mathrm{H} 2 \mathrm{~A}$ & 0.7659 & 0.9167 & 0.0595 & $0.024 *$ \\
\hline $\mathrm{H} 2 \mathrm{~B}$ & 0.5769 & 0.9607 & 0.0878 & $0.024^{*}$ \\
\hline $\mathrm{C} 3$ & $0.6802(2)$ & $0.83838(13)$ & 0.23985 (12) & $0.0187(3)$ \\
\hline $\mathrm{C} 4$ & $0.8705(2)$ & $0.87285(13)$ & $0.25612(12)$ & $0.0204(3)$ \\
\hline $\mathrm{C} 5$ & $0.9381(2)$ & $0.89174(14)$ & $0.35051(13)$ & $0.0229(4)$ \\
\hline H5 & 1.0668 & 0.9150 & 0.3628 & $0.028 *$ \\
\hline C6 & $0.8188(2)$ & $0.87689(14)$ & $0.42708(12)$ & $0.0224(3)$ \\
\hline H6 & 0.8679 & 0.8896 & 0.4909 & $0.027^{*}$ \\
\hline $\mathrm{C} 7$ & $0.6289(2)$ & $0.84384(13)$ & $0.41224(12)$ & $0.0193(3)$ \\
\hline $\mathrm{C} 8$ & $0.5638(2)$ & $0.82427(13)$ & $0.31696(12)$ & $0.0197(3)$ \\
\hline H8 & 0.4352 & 0.8005 & 0.3047 & $0.024 *$ \\
\hline $\mathrm{C} 9$ & $0.4964(2)$ & $0.83521(13)$ & $0.49259(12)$ & $0.0195(3)$ \\
\hline $\mathrm{C} 10$ & $0.5441(2)$ & $0.88953(14)$ & $0.58384(12)$ & 0.0223 \\
\hline
\end{tabular}
An approximate (isotropic) treatment of cell e.s.d.'s is used for estimating e.s.d.'s involving 1.s. planes.

Refinement. Maximum electron density of 0.56 e is in the vicinity of C 21 in the extended chain and may represent very minor disorder.

Fractional atomic coordinates and isotropic or equivalent isotropic displacement parameters $\left(\AA^{2}\right)$ 


$\begin{array}{lllll}\text { H10 } & 0.6661 & 0.9293 & 0.5958 & 0.027^{*} \\ \text { C11 } & 0.4178(2) & 0.88685(14) & 0.65740(12) & 0.0230(4) \\ \text { H11 } & 0.4549 & 0.9235 & 0.7190 & 0.028^{*} \\ \text { C12 } & 0.2375(2) & 0.83098(13) & 0.64171(12) & 0.0204(3) \\ \text { C13 } & 0.1889(3) & 0.77461(16) & 0.55146(13) & 0.0282(4) \\ \text { H13 } & 0.0665 & 0.7354 & 0.5396 & 0.034^{*} \\ \text { C14 } & 0.3165(3) & 0.77503(16) & 0.47882(13) & 0.0281(4) \\ \text { H14 } & 0.2816 & 0.7338 & 0.4187 & 0.034^{*} \\ \text { C15 } & 1.1677(3) & 0.92460(19) & 0.18946(15) & 0.0334(4) \\ \text { H15A } & 1.1870 & 0.9942 & 0.2319 & 0.050^{*} \\ \text { H15B } & 1.2233 & 0.9383 & 0.1272 & 0.050^{*} \\ \text { H15C } & 1.2255 & 0.8687 & 0.2185 & 0.050^{*} \\ \text { C16 } & 0.0893(2) & 0.82919(14) & 0.71575(13) & 0.0226(4) \\ \text { C17 } & 0.0153(3) & 0.82894(15) & 0.88628(13) & 0.0270(4) \\ \text { H17A } & -0.1093 & 0.8332 & 0.8612 & 0.032^{*} \\ \text { H17B } & 0.0500 & 0.8914 & 0.9369 & 0.032^{*} \\ \text { C18 } & 0.0089(3) & 0.71993(16) & 0.92983(13) & 0.0273(4) \\ \text { H18A } & 0.1319 & 0.7157 & 0.9570 & 0.033^{*} \\ \text { H18B } & -0.0813 & 0.7146 & 0.9822 & 0.033^{*} \\ \text { C19 } & 0.0108(3) & 0.52875(16) & 0.87786(14) & 0.0294(4) \\ \text { H19A } & -0.0770 & 0.4912 & 0.9229 & 0.035^{*} \\ \text { H19B } & 0.1350 & 0.5449 & 0.9090 & 0.035^{*} \\ \text { C20 } & 0.0145(3) & 0.45443(15) & 0.78734(15) & 0.0303(4) \\ \text { H20A } & 0.0284 & 0.3787 & 0.8026 & 0.036^{*} \\ \text { H20B } & -0.1026 & 0.4487 & 0.7505 & 0.036^{*} \\ \text { C21 } & 0.1833(3) & 0.42988(19) & 0.64803(17) & 0.0431(5) \\ \text { H21A } & 0.0660 & 0.4151 & 0.6101 & 0.052^{*} \\ \text { H21B } & 0.2117 & 0.3577 & 0.6651 & 0.052^{*} \\ \text { C22 } & 0.3357(4) & 0.4859(2) & 0.58984(17) & 0.0510(6) \\ \text { H22A } & 0.3335 & 0.4440 & 0.5256 & 0.061^{*} \\ \text { H22B } & 0.3141 & 0.5621 & 0.5807 & 0.061^{*} \\ \text { C23 } & 0.6108(3) & 0.60309(16) & 0.65657(15) & 0.0344(4) \\ \text { H23A } & 0.5972 & 0.6482 & 0.6026 & 0.041^{*} \\ \text { H23B } & 0.7437 & 0.6005 & 0.6651 & 0.041^{*} \\ \text { C24 } & 0.5416(3) & 0.65799(15) & 0.74680(14) & 0.0297(4) \\ \text { H24A } & 0.6178 & 0.7324 & 0.7631 & 0.036^{*} \\ \text { H24B } & 0.4123 & 0.6680 & 0.7366 & 0.036^{*} \\ & & & & \end{array}$

Atomic displacement parameters $\left(\AA^{2}\right)$

\begin{tabular}{lllllll}
\hline & $U^{11}$ & $U^{22}$ & $U^{33}$ & $U^{12}$ & $U^{13}$ & $U^{23}$ \\
\hline S1 & $0.0198(2)$ & $0.0170(2)$ & $0.0207(2)$ & $-0.00143(15)$ & $-0.00151(15)$ & $0.00555(14)$ \\
O1 & $0.0350(8)$ & $0.0252(6)$ & $0.0340(7)$ & $0.0097(5)$ & $0.0005(6)$ & $0.0085(5)$ \\
O2 & $0.0250(7)$ & $0.0316(7)$ & $0.0353(7)$ & $-0.0080(5)$ & $0.0031(6)$ & $0.0098(6)$ \\
O3 & $0.0334(7)$ & $0.0249(6)$ & $0.0221(6)$ & $0.0042(5)$ & $-0.0041(5)$ & $0.0077(5)$ \\
O4 & $0.0183(6)$ & $0.0312(6)$ & $0.0231(6)$ & $0.0026(5)$ & $0.0036(5)$ & $0.0060(5)$ \\
O5 & $0.0197(7)$ & $0.0391(7)$ & $0.0312(7)$ & $0.0057(5)$ & $0.0018(5)$ & $0.0076(6)$ \\
O6 & $0.0264(7)$ & $0.0275(6)$ & $0.0308(7)$ & $0.0055(5)$ & $0.0002(5)$ & $0.0065(5)$
\end{tabular}




\begin{tabular}{|c|c|c|c|c|c|c|}
\hline O7 & $0.0299(7)$ & $0.0246(6)$ & $0.0351(7)$ & $0.0004(5)$ & $0.0045(6)$ & $0.0007(5)$ \\
\hline $\mathrm{O} 8$ & $0.0422(8)$ & $0.0267(7)$ & $0.0368(7)$ & $0.0029(6)$ & $0.0066(6)$ & $-0.0014(6)$ \\
\hline N1 & $0.0289(8)$ & $0.0204(7)$ & $0.0217(7)$ & $0.0008(6)$ & $-0.0043(6)$ & $0.0044(6)$ \\
\hline N2 & $0.0275(8)$ & $0.0182(7)$ & $0.0188(7)$ & $-0.0036(5)$ & $-0.0031(6)$ & $0.0064(5)$ \\
\hline N3 & $0.0194(8)$ & $0.0262(8)$ & $0.0237(7)$ & $0.0021(6)$ & $0.0040(6)$ & $0.0032(6)$ \\
\hline N4 & $0.0342(10)$ & $0.0243(8)$ & $0.0300(8)$ & $0.0068(7)$ & $-0.0004(7)$ & $0.0049(6)$ \\
\hline $\mathrm{C} 1$ & $0.0199(8)$ & $0.0219(8)$ & $0.0206(8)$ & $0.0042(6)$ & $-0.0002(6)$ & $0.0039(6)$ \\
\hline $\mathrm{C} 2$ & $0.0218(8)$ & $0.0176(7)$ & $0.0201(8)$ & $0.0006(6)$ & $-0.0006(6)$ & $0.0069(6)$ \\
\hline C3 & $0.0212(8)$ & $0.0158(7)$ & $0.0188(8)$ & 0.0015 (6) & $-0.0018(6)$ & $0.0042(6)$ \\
\hline $\mathrm{C} 4$ & $0.0214(9)$ & $0.0180(7)$ & $0.0226(8)$ & $0.0035(6)$ & $0.0029(7)$ & $0.0049(6)$ \\
\hline $\mathrm{C} 5$ & $0.0166(8)$ & $0.0252(8)$ & $0.0263(9)$ & $0.0009(6)$ & $-0.0020(7)$ & $0.0035(7)$ \\
\hline C6 & $0.0219(9)$ & $0.0237(8)$ & $0.0212(8)$ & $0.0023(6)$ & $-0.0033(7)$ & $0.0026(6)$ \\
\hline $\mathrm{C} 7$ & $0.0206(9)$ & $0.0174(7)$ & $0.0205(8)$ & $0.0031(6)$ & $0.0002(6)$ & $0.0050(6)$ \\
\hline $\mathrm{C} 8$ & $0.0176(8)$ & $0.0189(8)$ & $0.0225(8)$ & $0.0015(6)$ & $-0.0012(6)$ & $0.0051(6)$ \\
\hline C9 & $0.0202(9)$ & $0.0188(8)$ & $0.0207(8)$ & $0.0039(6)$ & $-0.0003(6)$ & $0.0065(6)$ \\
\hline $\mathrm{C} 10$ & $0.0200(9)$ & $0.0226(8)$ & $0.0230(8)$ & $-0.0006(6)$ & $-0.0004(7)$ & $0.0031(6)$ \\
\hline C11 & $0.0266(9)$ & $0.0205(8)$ & $0.0210(8)$ & $0.0014(6)$ & $0.0001(7)$ & 0.0019 (6) \\
\hline $\mathrm{C} 12$ & $0.0216(9)$ & $0.0201(8)$ & $0.0212(8)$ & $0.0052(6)$ & $0.0012(6)$ & $0.0072(6)$ \\
\hline $\mathrm{C} 13$ & $0.0204(9)$ & $0.0373(10)$ & $0.0251(9)$ & $-0.0017(7)$ & $-0.0024(7)$ & $0.0052(7)$ \\
\hline $\mathrm{C} 14$ & $0.0251(10)$ & $0.0365(10)$ & $0.0202(8)$ & $-0.0020(7)$ & $-0.0017(7)$ & $0.0019(7)$ \\
\hline $\mathrm{C} 15$ & $0.0175(9)$ & $0.0517(12)$ & $0.0328(10)$ & $0.0062(8)$ & $0.0031(8)$ & $0.0133(9)$ \\
\hline $\mathrm{C} 16$ & $0.0229(10)$ & $0.0196(8)$ & $0.0258(9)$ & $0.0033(6)$ & $0.0030(7)$ & $0.0053(6)$ \\
\hline $\mathrm{C} 17$ & $0.0255(9)$ & $0.0293(9)$ & $0.0252(9)$ & $0.0032(7)$ & $0.0081(7)$ & $0.0006(7)$ \\
\hline $\mathrm{C} 18$ & $0.0241(9)$ & $0.0340(10)$ & $0.0231(8)$ & $0.0022(7)$ & $0.0040(7)$ & $0.0038(7)$ \\
\hline C19 & $0.0231(9)$ & $0.0292(9)$ & $0.0367(10)$ & $0.0016(7)$ & $-0.0006(8)$ & $0.0131(8)$ \\
\hline $\mathrm{C} 20$ & $0.0226(9)$ & $0.0249(9)$ & $0.0438(11)$ & $0.0018(7)$ & $0.0007(8)$ & $0.0094(8)$ \\
\hline $\mathrm{C} 21$ & $0.0386(12)$ & $0.0417(12)$ & $0.0427(12)$ & $-0.0026(9)$ & $0.0010(10)$ & $-0.0108(10)$ \\
\hline $\mathrm{C} 22$ & $0.0471(14)$ & $0.0703(17)$ & 0.0295 (11) & $-0.0007(12)$ & $0.0019(10)$ & $-0.0079(10)$ \\
\hline $\mathrm{C} 23$ & $0.0421(12)$ & $0.0263(9)$ & $0.0336(10)$ & $0.0014(8)$ & $0.0054(9)$ & $0.0049(8)$ \\
\hline C24 & $0.0369(11)$ & $0.0225(9)$ & $0.0296(9)$ & $0.0024(7)$ & $0.0016(8)$ & $0.0065(7)$ \\
\hline
\end{tabular}

Geometric parameters $\left(\AA,{ }^{\circ}\right)$

\begin{tabular}{llll}
\hline $\mathrm{S} 1-\mathrm{O} 2$ & $1.4341(13)$ & $\mathrm{C} 8-\mathrm{H} 8$ & 0.9500 \\
$\mathrm{~S} 1-\mathrm{O} 1$ & $1.4429(13)$ & $\mathrm{C} 9-\mathrm{C} 10$ & $1.397(2)$ \\
$\mathrm{S} 1-\mathrm{N} 1$ & $1.6025(14)$ & $\mathrm{C} 9-\mathrm{C} 14$ & $1.402(3)$ \\
$\mathrm{S} 1-\mathrm{N} 2$ & $1.6429(14)$ & $\mathrm{C} 10-\mathrm{C} 11$ & $1.388(2)$ \\
$\mathrm{O} 3-\mathrm{C} 1$ & $1.237(2)$ & $\mathrm{C} 10-\mathrm{H} 10$ & 0.9500 \\
$\mathrm{O} 4-\mathrm{C} 4$ & $1.365(2)$ & $\mathrm{C} 11-\mathrm{C} 12$ & $1.390(2)$ \\
$\mathrm{O} 4-\mathrm{C} 15$ & $1.425(2)$ & $\mathrm{C} 11-\mathrm{H} 11$ & 0.9500 \\
$\mathrm{O} 5-\mathrm{C} 16$ & $1.226(2)$ & $\mathrm{C} 12-\mathrm{C} 13$ & $1.395(3)$ \\
$\mathrm{O} 6-\mathrm{C} 19$ & $1.428(2)$ & $\mathrm{C} 12-\mathrm{C} 16$ & $1.505(2)$ \\
$\mathrm{O} 6-\mathrm{C} 18$ & $1.435(2)$ & $\mathrm{C} 13-\mathrm{C} 14$ & $1.386(3)$ \\
$\mathrm{O} 7-\mathrm{C} 21$ & $1.410(2)$ & $\mathrm{C} 13-\mathrm{H} 13$ & 0.9500 \\
$\mathrm{O} 7-\mathrm{C} 20$ & $1.414(2)$ & $\mathrm{C} 14-\mathrm{H} 14$ & 0.9500 \\
$\mathrm{O} 8-\mathrm{C} 22$ & $1.419(3)$ & $\mathrm{C} 15-\mathrm{H} 15 \mathrm{~A}$ & 0.9800 \\
$\mathrm{O} 8-\mathrm{C} 23$ & $1.424(2)$ & $\mathrm{C} 15-\mathrm{H} 15 \mathrm{~B}$ & 0.9800 \\
$\mathrm{~N} 1-\mathrm{C} 1$ & $1.345(2)$ & $\mathrm{C} 15-\mathrm{H} 15 \mathrm{C}$ & 0.9800
\end{tabular}




\begin{tabular}{|c|c|c|c|}
\hline $\mathrm{N} 2-\mathrm{C} 3$ & $1.425(2)$ & $\mathrm{C} 17-\mathrm{C} 18$ & $1.509(3)$ \\
\hline $\mathrm{N} 2-\mathrm{C} 2$ & $1.454(2)$ & $\mathrm{C} 17-\mathrm{H} 17 \mathrm{~A}$ & 0.9900 \\
\hline $\mathrm{N} 3-\mathrm{C} 16$ & $1.351(2)$ & C17-H17B & 0.9900 \\
\hline $\mathrm{N} 3-\mathrm{C} 17$ & $1.459(2)$ & $\mathrm{C} 18-\mathrm{H} 18 \mathrm{~A}$ & 0.9900 \\
\hline $\mathrm{N} 3-\mathrm{H} 1 \mathrm{~N} 3$ & $0.82(2)$ & $\mathrm{C} 18-\mathrm{H} 18 \mathrm{~B}$ & 0.9900 \\
\hline $\mathrm{N} 4-\mathrm{C} 24$ & $1.481(2)$ & $\mathrm{C} 19-\mathrm{C} 20$ & $1.491(3)$ \\
\hline $\mathrm{N} 4-\mathrm{H} 1 \mathrm{~N} 4$ & $0.93(3)$ & C19-H19A & 0.9900 \\
\hline $\mathrm{N} 4-\mathrm{H} 2 \mathrm{~N} 4$ & $1.03(3)$ & C19-H19B & 0.9900 \\
\hline $\mathrm{N} 4-\mathrm{H} 3 \mathrm{~N} 4$ & $0.98(3)$ & $\mathrm{C} 20-\mathrm{H} 20 \mathrm{~A}$ & 0.9900 \\
\hline $\mathrm{C} 1-\mathrm{C} 2$ & $1.515(2)$ & $\mathrm{C} 20-\mathrm{H} 20 \mathrm{~B}$ & 0.9900 \\
\hline $\mathrm{C} 2-\mathrm{H} 2 \mathrm{~A}$ & 0.9900 & $\mathrm{C} 21-\mathrm{C} 22$ & $1.496(3)$ \\
\hline $\mathrm{C} 2-\mathrm{H} 2 \mathrm{~B}$ & 0.9900 & $\mathrm{C} 21-\mathrm{H} 21 \mathrm{~A}$ & 0.9900 \\
\hline $\mathrm{C} 3-\mathrm{C} 8$ & $1.385(2)$ & $\mathrm{C} 21-\mathrm{H} 21 \mathrm{~B}$ & 0.9900 \\
\hline $\mathrm{C} 3-\mathrm{C} 4$ & $1.401(2)$ & $\mathrm{C} 22-\mathrm{H} 22 \mathrm{~A}$ & 0.9900 \\
\hline $\mathrm{C} 4-\mathrm{C} 5$ & $1.393(2)$ & $\mathrm{C} 22-\mathrm{H} 22 \mathrm{~B}$ & 0.9900 \\
\hline $\mathrm{C} 5-\mathrm{C} 6$ & $1.393(2)$ & $\mathrm{C} 23-\mathrm{C} 24$ & $1.505(3)$ \\
\hline $\mathrm{C} 5-\mathrm{H} 5$ & 0.9500 & $\mathrm{C} 23-\mathrm{H} 23 \mathrm{~A}$ & 0.9900 \\
\hline $\mathrm{C} 6-\mathrm{C} 7$ & $1.394(2)$ & $\mathrm{C} 23-\mathrm{H} 23 \mathrm{~B}$ & 0.9900 \\
\hline $\mathrm{C} 6-\mathrm{H} 6$ & 0.9500 & $\mathrm{C} 24-\mathrm{H} 24 \mathrm{~A}$ & 0.9900 \\
\hline $\mathrm{C} 7-\mathrm{C} 8$ & $1.399(2)$ & $\mathrm{C} 24-\mathrm{H} 24 \mathrm{~B}$ & 0.9900 \\
\hline $\mathrm{C} 7-\mathrm{C} 9$ & $1.490(2)$ & & \\
\hline $\mathrm{O} 2-\mathrm{S} 1-\mathrm{O} 1$ & $113.21(8)$ & $\mathrm{C} 14-\mathrm{C} 13-\mathrm{H} 13$ & 119.5 \\
\hline $\mathrm{O} 2-\mathrm{S} 1-\mathrm{N} 1$ & $112.24(8)$ & $\mathrm{C} 12-\mathrm{C} 13-\mathrm{H} 13$ & 119.5 \\
\hline $\mathrm{O} 1-\mathrm{S} 1-\mathrm{N} 1$ & $111.49(8)$ & $\mathrm{C} 13-\mathrm{C} 14-\mathrm{C} 9$ & $121.12(17)$ \\
\hline $\mathrm{O} 2-\mathrm{S} 1-\mathrm{N} 2$ & $109.69(8)$ & $\mathrm{C} 13-\mathrm{C} 14-\mathrm{H} 14$ & 119.4 \\
\hline $\mathrm{O} 1-\mathrm{S} 1-\mathrm{N} 2$ & $111.90(8)$ & $\mathrm{C} 9-\mathrm{C} 14-\mathrm{H} 14$ & 119.4 \\
\hline $\mathrm{N} 1-\mathrm{S} 1-\mathrm{N} 2$ & $97.26(7)$ & $\mathrm{O} 4-\mathrm{C} 15-\mathrm{H} 15 \mathrm{~A}$ & 109.5 \\
\hline $\mathrm{C} 4-\mathrm{O} 4-\mathrm{C} 15$ & $117.63(14)$ & $\mathrm{O} 4-\mathrm{C} 15-\mathrm{H} 15 \mathrm{~B}$ & 109.5 \\
\hline $\mathrm{C} 19-\mathrm{O} 6-\mathrm{C} 18$ & $112.83(14)$ & $\mathrm{H} 15 \mathrm{~A}-\mathrm{C} 15-\mathrm{H} 15 \mathrm{~B}$ & 109.5 \\
\hline $\mathrm{C} 21-\mathrm{O} 7-\mathrm{C} 20$ & $111.72(15)$ & $\mathrm{O} 4-\mathrm{C} 15-\mathrm{H} 15 \mathrm{C}$ & 109.5 \\
\hline $\mathrm{C} 22-\mathrm{O} 8-\mathrm{C} 23$ & $115.10(18)$ & $\mathrm{H} 15 \mathrm{~A}-\mathrm{C} 15-\mathrm{H} 15 \mathrm{C}$ & 109.5 \\
\hline $\mathrm{C} 1-\mathrm{N} 1-\mathrm{S} 1$ & $111.85(12)$ & $\mathrm{H} 15 \mathrm{~B}-\mathrm{C} 15-\mathrm{H} 15 \mathrm{C}$ & 109.5 \\
\hline $\mathrm{C} 3-\mathrm{N} 2-\mathrm{C} 2$ & $125.75(13)$ & $\mathrm{O} 5-\mathrm{C} 16-\mathrm{N} 3$ & $123.27(16)$ \\
\hline $\mathrm{C} 3-\mathrm{N} 2-\mathrm{S} 1$ & $120.81(11)$ & $\mathrm{O} 5-\mathrm{C} 16-\mathrm{C} 12$ & $120.31(16)$ \\
\hline $\mathrm{C} 2-\mathrm{N} 2-\mathrm{S} 1$ & $111.22(11)$ & $\mathrm{N} 3-\mathrm{C} 16-\mathrm{C} 12$ & $116.41(16)$ \\
\hline $\mathrm{C} 16-\mathrm{N} 3-\mathrm{C} 17$ & $121.27(16)$ & $\mathrm{N} 3-\mathrm{C} 17-\mathrm{C} 18$ & $112.19(15)$ \\
\hline $\mathrm{C} 16-\mathrm{N} 3-\mathrm{H} 1 \mathrm{~N} 3$ & $120.7(16)$ & $\mathrm{N} 3-\mathrm{C} 17-\mathrm{H} 17 \mathrm{~A}$ & 109.2 \\
\hline $\mathrm{C} 17-\mathrm{N} 3-\mathrm{H} 1 \mathrm{~N} 3$ & $116.9(15)$ & $\mathrm{C} 18-\mathrm{C} 17-\mathrm{H} 17 \mathrm{~A}$ & 109.2 \\
\hline $\mathrm{C} 24-\mathrm{N} 4-\mathrm{H} 1 \mathrm{~N} 4$ & $108.5(17)$ & $\mathrm{N} 3-\mathrm{C} 17-\mathrm{H} 17 \mathrm{~B}$ & 109.2 \\
\hline $\mathrm{C} 24-\mathrm{N} 4-\mathrm{H} 2 \mathrm{~N} 4$ & $113.3(14)$ & $\mathrm{C} 18-\mathrm{C} 17-\mathrm{H} 17 \mathrm{~B}$ & 109.2 \\
\hline $\mathrm{H} 1 \mathrm{~N} 4-\mathrm{N} 4-\mathrm{H} 2 \mathrm{~N} 4$ & $107(2)$ & $\mathrm{H} 17 \mathrm{~A}-\mathrm{C} 17-\mathrm{H} 17 \mathrm{~B}$ & 107.9 \\
\hline $\mathrm{C} 24-\mathrm{N} 4-\mathrm{H} 3 \mathrm{~N} 4$ & $102.2(17)$ & $\mathrm{O} 6-\mathrm{C} 18-\mathrm{C} 17$ & $108.55(14)$ \\
\hline $\mathrm{H} 1 \mathrm{~N} 4-\mathrm{N} 4-\mathrm{H} 3 \mathrm{~N} 4$ & $117(2)$ & $\mathrm{O} 6-\mathrm{C} 18-\mathrm{H} 18 \mathrm{~A}$ & 110.0 \\
\hline $\mathrm{H} 2 \mathrm{~N} 4-\mathrm{N} 4-\mathrm{H} 3 \mathrm{~N} 4$ & $109(2)$ & $\mathrm{C} 17-\mathrm{C} 18-\mathrm{H} 18 \mathrm{~A}$ & 110.0 \\
\hline $\mathrm{O} 3-\mathrm{C} 1-\mathrm{N} 1$ & $124.30(16)$ & $\mathrm{O} 6-\mathrm{C} 18-\mathrm{H} 18 \mathrm{~B}$ & 110.0 \\
\hline $\mathrm{O} 3-\mathrm{C} 1-\mathrm{C} 2$ & $121.76(15)$ & $\mathrm{C} 17-\mathrm{C} 18-\mathrm{H} 18 \mathrm{~B}$ & 110.0 \\
\hline $\mathrm{N} 1-\mathrm{C} 1-\mathrm{C} 2$ & $113.94(14)$ & $\mathrm{H} 18 \mathrm{~A}-\mathrm{C} 18-\mathrm{H} 18 \mathrm{~B}$ & 108.4 \\
\hline
\end{tabular}




\begin{tabular}{|c|c|}
\hline $\mathrm{N} 2-\mathrm{C} 2-\mathrm{C} 1$ & $104.42(13)$ \\
\hline $\mathrm{N} 2-\mathrm{C} 2-\mathrm{H} 2 \mathrm{~A}$ & 110.9 \\
\hline $\mathrm{C} 1-\mathrm{C} 2-\mathrm{H} 2 \mathrm{~A}$ & 110.9 \\
\hline $\mathrm{N} 2-\mathrm{C} 2-\mathrm{H} 2 \mathrm{~B}$ & 110.9 \\
\hline $\mathrm{C} 1-\mathrm{C} 2-\mathrm{H} 2 \mathrm{~B}$ & 110.9 \\
\hline $\mathrm{H} 2 \mathrm{~A}-\mathrm{C} 2-\mathrm{H} 2 \mathrm{~B}$ & 108.9 \\
\hline $\mathrm{C} 8-\mathrm{C} 3-\mathrm{C} 4$ & $119.87(15)$ \\
\hline $\mathrm{C} 8-\mathrm{C} 3-\mathrm{N} 2$ & $118.94(15)$ \\
\hline $\mathrm{C} 4-\mathrm{C} 3-\mathrm{N} 2$ & $121.19(15)$ \\
\hline $\mathrm{O} 4-\mathrm{C} 4-\mathrm{C} 5$ & $125.49(16)$ \\
\hline $\mathrm{O} 4-\mathrm{C} 4-\mathrm{C} 3$ & $115.86(15)$ \\
\hline $\mathrm{C} 5-\mathrm{C} 4-\mathrm{C} 3$ & $118.65(15)$ \\
\hline $\mathrm{C} 6-\mathrm{C} 5-\mathrm{C} 4$ & $120.62(16)$ \\
\hline $\mathrm{C} 6-\mathrm{C} 5-\mathrm{H} 5$ & 119.7 \\
\hline $\mathrm{C} 4-\mathrm{C} 5-\mathrm{H} 5$ & 119.7 \\
\hline $\mathrm{C} 5-\mathrm{C} 6-\mathrm{C} 7$ & $121.50(16)$ \\
\hline $\mathrm{C} 5-\mathrm{C} 6-\mathrm{H} 6$ & 119.3 \\
\hline $\mathrm{C} 7-\mathrm{C} 6-\mathrm{H} 6$ & 119.3 \\
\hline $\mathrm{C} 6-\mathrm{C} 7-\mathrm{C} 8$ & $117.06(15)$ \\
\hline $\mathrm{C} 6-\mathrm{C} 7-\mathrm{C} 9$ & $122.74(15)$ \\
\hline $\mathrm{C} 8-\mathrm{C} 7-\mathrm{C} 9$ & $120.11(15)$ \\
\hline $\mathrm{C} 3-\mathrm{C} 8-\mathrm{C} 7$ & $122.29(16)$ \\
\hline $\mathrm{C} 3-\mathrm{C} 8-\mathrm{H} 8$ & 118.9 \\
\hline $\mathrm{C} 7-\mathrm{C} 8-\mathrm{H} 8$ & 118.9 \\
\hline $\mathrm{C} 10-\mathrm{C} 9-\mathrm{C} 14$ & $117.26(16)$ \\
\hline $\mathrm{C} 10-\mathrm{C} 9-\mathrm{C} 7$ & $121.45(15)$ \\
\hline $\mathrm{C} 14-\mathrm{C} 9-\mathrm{C} 7$ & $121.26(15)$ \\
\hline $\mathrm{C} 11-\mathrm{C} 10-\mathrm{C} 9$ & $121.63(16)$ \\
\hline $\mathrm{C} 11-\mathrm{C} 10-\mathrm{H} 10$ & 119.2 \\
\hline $\mathrm{C} 9-\mathrm{C} 10-\mathrm{H} 10$ & 119.2 \\
\hline $\mathrm{C} 10-\mathrm{C} 11-\mathrm{C} 12$ & $120.59(16)$ \\
\hline $\mathrm{C} 10-\mathrm{C} 11-\mathrm{H} 11$ & 119.7 \\
\hline $\mathrm{C} 12-\mathrm{C} 11-\mathrm{H} 11$ & 119.7 \\
\hline $\mathrm{C} 11-\mathrm{C} 12-\mathrm{C} 13$ & $118.37(16)$ \\
\hline $\mathrm{C} 11-\mathrm{C} 12-\mathrm{C} 16$ & $123.98(16)$ \\
\hline $\mathrm{C} 13-\mathrm{C} 12-\mathrm{C} 16$ & $117.63(16)$ \\
\hline $\mathrm{C} 14-\mathrm{C} 13-\mathrm{C} 12$ & $120.93(17)$ \\
\hline $\mathrm{O} 2-\mathrm{S} 1-\mathrm{N} 1-\mathrm{C} 1$ & $121.10(13)$ \\
\hline $\mathrm{O} 1-\mathrm{S} 1-\mathrm{N} 1-\mathrm{C} 1$ & $-110.68(13)$ \\
\hline $\mathrm{N} 2-\mathrm{S} 1-\mathrm{N} 1-\mathrm{C} 1$ & $6.33(14)$ \\
\hline $\mathrm{O} 2-\mathrm{S} 1-\mathrm{N} 2-\mathrm{C} 3$ & $68.27(15)$ \\
\hline $\mathrm{O} 1-\mathrm{S} 1-\mathrm{N} 2-\mathrm{C} 3$ & $-58.24(15)$ \\
\hline $\mathrm{N} 1-\mathrm{S} 1-\mathrm{N} 2-\mathrm{C} 3$ & $-174.93(14)$ \\
\hline $\mathrm{O} 2-\mathrm{S} 1-\mathrm{N} 2-\mathrm{C} 2$ & $-127.73(12)$ \\
\hline $\mathrm{O} 1-\mathrm{S} 1-\mathrm{N} 2-\mathrm{C} 2$ & $105.76(13)$ \\
\hline $\mathrm{N} 1-\mathrm{S} 1-\mathrm{N} 2-\mathrm{C} 2$ & $-10.94(13)$ \\
\hline $\mathrm{S} 1-\mathrm{N} 1-\mathrm{C} 1-\mathrm{O} 3$ & $179.31(14)$ \\
\hline
\end{tabular}

\begin{tabular}{|c|c|}
\hline $\mathrm{O} 6-\mathrm{C} 19-\mathrm{C} 20$ & $109.31(15)$ \\
\hline $\mathrm{O} 6-\mathrm{C} 19-\mathrm{H} 19 \mathrm{~A}$ & 109.8 \\
\hline $\mathrm{C} 20-\mathrm{C} 19-\mathrm{H} 19 \mathrm{~A}$ & 109.8 \\
\hline $\mathrm{O} 6-\mathrm{C} 19-\mathrm{H} 19 \mathrm{~B}$ & 109.8 \\
\hline $\mathrm{C} 20-\mathrm{C} 19-\mathrm{H} 19 \mathrm{~B}$ & 109.8 \\
\hline $\mathrm{H} 19 \mathrm{~A}-\mathrm{C} 19-\mathrm{H} 19 \mathrm{~B}$ & 108.3 \\
\hline $\mathrm{O} 7-\mathrm{C} 20-\mathrm{C} 19$ & $108.74(15)$ \\
\hline $\mathrm{O} 7-\mathrm{C} 20-\mathrm{H} 20 \mathrm{~A}$ & 109.9 \\
\hline $\mathrm{C} 19-\mathrm{C} 20-\mathrm{H} 20 \mathrm{~A}$ & 109.9 \\
\hline $\mathrm{O} 7-\mathrm{C} 20-\mathrm{H} 20 \mathrm{~B}$ & 109.9 \\
\hline $\mathrm{C} 19-\mathrm{C} 20-\mathrm{H} 20 \mathrm{~B}$ & 109.9 \\
\hline $\mathrm{H} 20 \mathrm{~A}-\mathrm{C} 20-\mathrm{H} 20 \mathrm{~B}$ & 108.3 \\
\hline $\mathrm{O} 7-\mathrm{C} 21-\mathrm{C} 22$ & $109.08(18)$ \\
\hline $\mathrm{O} 7-\mathrm{C} 21-\mathrm{H} 21 \mathrm{~A}$ & 109.9 \\
\hline $\mathrm{C} 22-\mathrm{C} 21-\mathrm{H} 21 \mathrm{~A}$ & 109.9 \\
\hline $\mathrm{O} 7-\mathrm{C} 21-\mathrm{H} 21 \mathrm{~B}$ & 109.9 \\
\hline $\mathrm{C} 22-\mathrm{C} 21-\mathrm{H} 21 \mathrm{~B}$ & 109.9 \\
\hline $\mathrm{H} 21 \mathrm{~A}-\mathrm{C} 21-\mathrm{H} 21 \mathrm{~B}$ & 108.3 \\
\hline $\mathrm{O} 8-\mathrm{C} 22-\mathrm{C} 21$ & $112.5(2)$ \\
\hline $\mathrm{O} 8-\mathrm{C} 22-\mathrm{H} 22 \mathrm{~A}$ & 109.1 \\
\hline $\mathrm{C} 21-\mathrm{C} 22-\mathrm{H} 22 \mathrm{~A}$ & 109.1 \\
\hline $\mathrm{O} 8-\mathrm{C} 22-\mathrm{H} 22 \mathrm{~B}$ & 109.1 \\
\hline $\mathrm{C} 21-\mathrm{C} 22-\mathrm{H} 22 \mathrm{~B}$ & 109.1 \\
\hline $\mathrm{H} 22 \mathrm{~A}-\mathrm{C} 22-\mathrm{H} 22 \mathrm{~B}$ & 107.8 \\
\hline $\mathrm{O} 8-\mathrm{C} 23-\mathrm{C} 24$ & $111.71(16)$ \\
\hline $\mathrm{O} 8-\mathrm{C} 23-\mathrm{H} 23 \mathrm{~A}$ & 109.3 \\
\hline $\mathrm{C} 24-\mathrm{C} 23-\mathrm{H} 23 \mathrm{~A}$ & 109.3 \\
\hline $\mathrm{O} 8-\mathrm{C} 23-\mathrm{H} 23 \mathrm{~B}$ & 109.3 \\
\hline $\mathrm{C} 24-\mathrm{C} 23-\mathrm{H} 23 \mathrm{~B}$ & 109.3 \\
\hline $\mathrm{H} 23 \mathrm{~A}-\mathrm{C} 23-\mathrm{H} 23 \mathrm{~B}$ & 107.9 \\
\hline $\mathrm{N} 4-\mathrm{C} 24-\mathrm{C} 23$ & $109.41(16)$ \\
\hline $\mathrm{N} 4-\mathrm{C} 24-\mathrm{H} 24 \mathrm{~A}$ & 109.8 \\
\hline $\mathrm{C} 23-\mathrm{C} 24-\mathrm{H} 24 \mathrm{~A}$ & 109.8 \\
\hline $\mathrm{N} 4-\mathrm{C} 24-\mathrm{H} 24 \mathrm{~B}$ & 109.8 \\
\hline $\mathrm{C} 23-\mathrm{C} 24-\mathrm{H} 24 \mathrm{~B}$ & 109.8 \\
\hline $\mathrm{H} 24 \mathrm{~A}-\mathrm{C} 24-\mathrm{H} 24 \mathrm{~B}$ & 108.2 \\
\hline $\mathrm{C} 9-\mathrm{C} 7-\mathrm{C} 8-\mathrm{C} 3$ & $175.66(14)$ \\
\hline $\mathrm{C} 6-\mathrm{C} 7-\mathrm{C} 9-\mathrm{C} 10$ & $19.1(2)$ \\
\hline $\mathrm{C} 8-\mathrm{C} 7-\mathrm{C} 9-\mathrm{C} 10$ & $-157.39(16)$ \\
\hline $\mathrm{C} 6-\mathrm{C} 7-\mathrm{C} 9-\mathrm{C} 14$ & $-162.95(16)$ \\
\hline $\mathrm{C} 8-\mathrm{C} 7-\mathrm{C} 9-\mathrm{C} 14$ & $20.6(2)$ \\
\hline $\mathrm{C} 14-\mathrm{C} 9-\mathrm{C} 10-\mathrm{C} 11$ & $-1.7(3)$ \\
\hline $\mathrm{C} 7-\mathrm{C} 9-\mathrm{C} 10-\mathrm{C} 11$ & $176.34(15)$ \\
\hline $\mathrm{C} 9-\mathrm{C} 10-\mathrm{C} 11-\mathrm{C} 12$ & $-1.1(3)$ \\
\hline $\mathrm{C} 10-\mathrm{C} 11-\mathrm{C} 12-\mathrm{C} 13$ & $2.2(2)$ \\
\hline $\mathrm{C} 10-\mathrm{C} 11-\mathrm{C} 12-\mathrm{C} 16$ & $-176.56(15)$ \\
\hline
\end{tabular}




$\begin{array}{llll}\mathrm{S} 1-\mathrm{N} 1-\mathrm{C} 1-\mathrm{C} 2 & 0.00(19) & \mathrm{C} 11-\mathrm{C} 12-\mathrm{C} 13-\mathrm{C} 14 & -0.6(3) \\ \mathrm{C} 3-\mathrm{N} 2-\mathrm{C} 2-\mathrm{C} 1 & 174.45(15) & \mathrm{C} 16-\mathrm{C} 12-\mathrm{C} 13-\mathrm{C} 14 & 178.28(17) \\ \mathrm{S} 1-\mathrm{N} 2-\mathrm{C} 2-\mathrm{C} 1 & 11.42(16) & \mathrm{C} 12-\mathrm{C} 13-\mathrm{C} 14-\mathrm{C} 9 & -2.3(3) \\ \mathrm{O} 3-\mathrm{C} 1-\mathrm{C} 2-\mathrm{N} 2 & 173.35(16) & \mathrm{C} 10-\mathrm{C} 9-\mathrm{C} 14-\mathrm{C} 13 & 3.4(3) \\ \mathrm{N} 1-\mathrm{C} 1-\mathrm{C} 2-\mathrm{N} 2 & -7.3(2) & \mathrm{C} 7-\mathrm{C} 9-\mathrm{C} 14-\mathrm{C} 13 & -174.70(17) \\ \mathrm{C} 2-\mathrm{N} 2-\mathrm{C} 3-\mathrm{C} 8 & 127.60(17) & \mathrm{C} 17-\mathrm{N} 3-\mathrm{C} 16-\mathrm{O} 5 & 7.3(3) \\ \mathrm{S} 1-\mathrm{N} 2-\mathrm{C} 3-\mathrm{C} 8 & -70.86(19) & \mathrm{C} 17-\mathrm{N} 3-\mathrm{C} 16-\mathrm{C} 12 & -173.40(14) \\ \mathrm{C} 2-\mathrm{N} 2-\mathrm{C} 3-\mathrm{C} 4 & -52.4(2) & \mathrm{C} 11-\mathrm{C} 12-\mathrm{C} 16-\mathrm{O} 5 & 151.68(17) \\ \mathrm{S} 1-\mathrm{N} 2-\mathrm{C} 3-\mathrm{C} 4 & 109.13(16) & \mathrm{C} 13-\mathrm{C} 12-\mathrm{C} 16-\mathrm{O} 5 & -27.1(2) \\ \mathrm{C} 15-\mathrm{O} 4-\mathrm{C} 4-\mathrm{C} 5 & -3.1(2) & \mathrm{C} 11-\mathrm{C} 12-\mathrm{C} 16-\mathrm{N} 3 & -27.6(2) \\ \mathrm{C} 15-\mathrm{O} 4-\mathrm{C} 4-\mathrm{C} 3 & 177.13(15) & \mathrm{C} 13-\mathrm{C} 12-\mathrm{C} 16-\mathrm{N} 3 & 153.56(16) \\ \mathrm{C} 8-\mathrm{C} 3-\mathrm{C} 4-\mathrm{O} 4 & -179.96(14) & \mathrm{C} 16-\mathrm{N} 3-\mathrm{C} 17-\mathrm{C} 18 & 105.25(19) \\ \mathrm{N} 2-\mathrm{C} 3-\mathrm{C} 4-\mathrm{O} 4 & 0.1(2) & \mathrm{C} 19-\mathrm{O}-\mathrm{C} 18-\mathrm{C} 17 & 157.75(15) \\ \mathrm{C} 8-\mathrm{C} 3-\mathrm{C} 4-\mathrm{C} 5 & 0.3(2) & \mathrm{N} 3-\mathrm{C} 17-\mathrm{C} 18-\mathrm{O} 6 & -59.8(2) \\ \mathrm{N} 2-\mathrm{C} 3-\mathrm{C} 4-\mathrm{C} 5 & -179.71(15) & \mathrm{C} 18-\mathrm{O}-\mathrm{C} 19-\mathrm{C} 20 & -159.42(15) \\ \mathrm{O} 4-\mathrm{C} 4-\mathrm{C} 5-\mathrm{C} 6 & -179.90(15) & \mathrm{C} 21-\mathrm{O} 7-\mathrm{C} 20-\mathrm{C} 19 & 176.42(17) \\ \mathrm{C} 3-\mathrm{C} 4-\mathrm{C} 5-\mathrm{C} 6 & -0.2(2) & \mathrm{O} 6-\mathrm{C} 19-\mathrm{C} 20-\mathrm{O} 7 & 70.69(19) \\ \mathrm{C} 4-\mathrm{C} 5-\mathrm{C} 6-\mathrm{C} 7 & -0.6(3) & \mathrm{C} 20-\mathrm{O} 7-\mathrm{C} 21-\mathrm{C} 22 & 176.10(19) \\ \mathrm{C} 5-\mathrm{C} 6-\mathrm{C} 7-\mathrm{C} 8 & 1.1(2) & \mathrm{C} 23-\mathrm{O} 8-\mathrm{C} 22-\mathrm{C} 21 & -119.2(2) \\ \mathrm{C} 5-\mathrm{C} 6-\mathrm{C} 7-\mathrm{C} 9 & -175.45(15) & \mathrm{O} 7-\mathrm{C} 21-\mathrm{C} 22-\mathrm{O} 8 & 69.3(3) \\ \mathrm{C} 4-\mathrm{C} 3-\mathrm{C} 8-\mathrm{C} 7 & 0.3(2) & \mathrm{C} 22-\mathrm{O} 8-\mathrm{C} 23-\mathrm{C} 24 & 78.5(2) \\ \mathrm{N} 2-\mathrm{C} 3-\mathrm{C} 8-\mathrm{C} 7 & -179.69(14) & \mathrm{O} 8-\mathrm{C} 23-\mathrm{C} 24-\mathrm{N} 4 & 55.8(2) \\ \mathrm{C} 6-\mathrm{C} 7-\mathrm{C} 8-\mathrm{C} 3 & -1.0(2) & & \\ & & & \end{array}$

Hydrogen-bond geometry $\left(A,{ }^{\circ}\right)$

$\mathrm{Cg} 1$ is the centroid of the $\mathrm{C} 3-\mathrm{C} 8$ ring.

\begin{tabular}{|c|c|c|c|c|}
\hline$D-\mathrm{H} \cdots A$ & $D-\mathrm{H}$ & $\mathrm{H} \cdots A$ & $D \cdots A$ & $D-\mathrm{H} \cdots A$ \\
\hline $\mathrm{N} 3-\mathrm{H} 1 N 3 \cdots 3^{\mathrm{i}}$ & $0.82(2)$ & $2.22(3)$ & $3.012(2)$ & $161(2)$ \\
\hline $\mathrm{N} 4-\mathrm{H} 1 N 4 \cdots \mathrm{O} 1^{\mathrm{ii}}$ & $0.93(3)$ & $2.29(3)$ & $3.010(2)$ & $133(2)$ \\
\hline $\mathrm{N} 4-\mathrm{H} 1 N 4 \cdots \mathrm{O} 7$ & $0.93(3)$ & $2.49(3)$ & $3.106(2)$ & $124(2)$ \\
\hline $\mathrm{N} 4-\mathrm{H} 2 N 4 \cdots \mathrm{N} 1^{\mathrm{i}}$ & $1.03(3)$ & $1.82(3)$ & $2.821(2)$ & $163(2)$ \\
\hline $\mathrm{N} 4-\mathrm{H} 3 N 4 \cdots \mathrm{O}^{\mathrm{iii}}$ & $0.98(3)$ & $1.99(3)$ & $2.942(2)$ & $162(3)$ \\
\hline $\mathrm{C} 2-\mathrm{H} 2 B \cdots \mathrm{O} 3^{\text {iv }}$ & 0.99 & 2.30 & $3.267(2)$ & 166 \\
\hline $\mathrm{C} 18-\mathrm{H} 18 A \cdots \mathrm{N} 1^{\mathrm{i}}$ & 0.99 & 2.57 & $3.545(2)$ & 168 \\
\hline $\mathrm{C} 22-\mathrm{H} 22 A \cdots \mathrm{O}^{\mathrm{ii}}$ & 0.99 & 2.63 & $3.343(3)$ & 129 \\
\hline $\mathrm{C} 24-\mathrm{H} 24 A \cdots \mathrm{O} 5^{\mathrm{iii}}$ & 0.99 & 2.58 & $3.298(2)$ & 129 \\
\hline $\mathrm{C} 21-\mathrm{H} 21 B \cdots C g 1^{\mathrm{ii}}$ & 0.99 & 2.70 & $3.555(2)$ & 165 \\
\hline
\end{tabular}

Symmetry codes: (i) $x, y, z+1$; (ii) $-x+1,-y+1,-z+1$; (iii) $x+1, y, z$; (iv) $-x+1,-y+2,-z$. 